\title{
Artery Stenosis of the Renal Graft: Experience of a Center of Northeastern Brazil
}

\author{
J.C.L. Nasserala ${ }^{a, \star}$, C.M.C. Oliveira ${ }^{a}$, J.B.G. Cerqueira ${ }^{a}$, S. Souza ${ }^{a}$, S.L. Silva ${ }^{a}$, L.C.d.O. Santos ${ }^{a}$,
} S.A. Andrade ${ }^{a}$, W.M. Barroso ${ }^{a}$, P.F.C.B.C. Fernandes ${ }^{a}$, A.G. Fernandes ${ }^{a}$, and E.F. Daher ${ }^{a}$ b

${ }^{a}$ Division of Nephrology, Hospital Universitário Walter Cantídio, Federal University of Ceara, Fortaleza, CE, Brazil; and ${ }^{\mathrm{b}}$ Department of Internal Medicine, Post-Graduation Program in Medical Sciences, Faculty of Medicine, Federal University of Ceará, Fortaleza, CE, Brazil

\begin{abstract}
Background. Transplant renal artery stenosis (TRAS), the most common vascular complication after transplant (Tx), leads to resistant hypertension, impaired renal function, and even loss of the graft. The purpose of the study was to investigate the prevalence and factors associated with TRAS in northeastern Brazil.

Methods. The study was conducted as a retrospective case-control study in a population of Tx recipients in a renal Tx center in northeastern Brazil. Demographic and clinical characteristics of the recipients and donors, data related to the surgery, laboratory data, and number of anti-hypertensive drugs were assessed. Statistical analysis was performed with the use of SPSS 17.0.

Results. A total of 494 of 529 recipients were assessed, of which 24 had TRAS. The prevalence of TRAS was $4.8 \%$. Twelve patients $(50 \%)$ were men with a mean age of $46.7 \pm$ 13.5 years. The mean time of diagnosis was 89.9 days after Tx. The risk factors associated with TRAS were number of anti-hypertensive drugs $\geq 2$ (odds ratio, 17.0; confidence interval, 4.1 to $70.4 ; P=.001$ ) and grafting with 2 or more arteries (odds ratio, 8.9; confidence interval, 1.4 to $56.6 ; P=.021)$. There was a significant reduction in mean systolic blood pressure $(147.1 \pm 23.7$ to $127.8 \pm 15.2 \mathrm{~mm} \mathrm{Hg}, P=.001)$ and diastolic blood pressure $(86.6 \pm 13.0$ to $77.6 \pm 9.4 \mathrm{~mm} \mathrm{Hg}, P=.001)$ after TRAS repair and in serum creatinine $(2.8 \pm 2.4$ to $1.9 \pm 1.8 \mathrm{mg} / \mathrm{dL}, P=.04)$.

Conclusions. Grafts with 2 or more arteries are associated with TRAS, as well as patients who use a higher number of anti-hypertensive drugs. TRAS repair was associated with improved blood pressure control and renal function.
\end{abstract}

$\mathbf{T}^{\mathrm{p}}$ RANSPLANT RENAL ARTERY STENOSIS (TRAS) is the most common vascular complication after transplantation (Tx), representing $75 \%$ of these complications. The incidence reported in the literature varies from $1 \%$ to $23 \%$, being an important cause of hypertension, renal function deterioration, and/or graft loss [1-11].

TRAS can be diagnosed at any time after renal transplantation, but it usually becomes apparent between the third month and the second year after Tx, being of multifactorial origin $[2,4,5]$. Regarding the location of the renal transplant arterial anastomosis, the stenosis may be proximal as the result of atherosclerotic disease in the recipient, at the anastomosis, or distal, in the donor's renal artery. Regarding stenosis involvement, it may be diffuse or multiple [11,12,31]. Early anastomosis stenosis is generally associated with trauma during surgery and/or postoperative fibrosis. The etiology of the distal TRAS is less clear but may be due to mechanical or immunological damage. Regardless of the stenosis location, early diagnosis is important to reduce morbidity and mortality [12].

The gold standard for the diagnosis of TRAS is still renal arteriography. Different, less invasive methods are available

*Address correspondence to Jarinne Camilo L. Nasserala, Rua General Vieira de Melo, No. 1412, Bairro Jardim Europa, CEP 69915-182, Rio Branco-Acre, Brazil. E-mail: jcInasserala@gmail. com 
to confirm the diagnosis, such as Doppler ultrasonography (US), magnetic resonance imaging, computed tomography, or scintigraphy with captopril. The use of these methods depends in part on the center's experience. The graft Doppler US is the test of choice for recipients with graft dysfunction [13-15].

TRAS treatment modalities include surgical and interventional radiological treatments. Percutaneous transluminal angioplasty (PTA), with or without stenting, is the main therapy because it is less invasive, with a reduced number of severe complications and little chance of failure. An adequate clinical response to PTA of renal artery stenosis is the return of renal function and blood pressure control $[4,16]$.

Technical success and complication rates of PTA of renal artery stenosis are $60 \%$ to $94 \%$ and $0 \%$ to $8.3 \%$, respectively, with graft loss rarely occurring [17]. Pillot et al [4] found $100 \%$ efficacy in TRAS repair with the endovascular procedure. Surgical treatment is reserved for patients with anastomosis stenosis or severe distal artery stenosis that is inaccessible through PTA $[5,17]$.

Consequently, because this is the most common vascular complication after renal transplantation and because there are few available data in Brazil, the aim of this study was to identify the prevalence and factors associated with TRAS in a referral center for kidney transplantation in northeastern Brazil.

\section{METHODS}

All patients submitted to renal transplantation at Hospital Universitário Walter Cantídio, Fortaleza, Ceará, Brazil, from January 2008 to March 2014 were included in the study, whereas those lost to follow-up because of being transferred to another renal transplant unit were excluded. A retrospective, case-control study was carried out with patients with suspected TRAS at the Doppler US. A control group of patients submitted to renal Tx who did not have TRAS was chosen in this transplant center in the same study period, being matched according to the type of donor and age/sex of the recipient and donor, with 2 control patients for each TRAS case.

The criteria used at the Doppler US to diagnose TRAS were peak systolic velocity (PSV) $>250 \mathrm{~cm} / \mathrm{s}$ and/or parvus-tardus waveform (acceleration time $>0.07$ seconds, acceleration index $<3 \mathrm{~m} / \mathrm{s}^{2}$ ) and intrarenal artery pattern represented by the Pourcelot resistive index (RI; normal range, 0.5 to 0.7 ), which is the result of dividing the difference at the maximum PSV and minimum diastolic velocity (MDV) by the maximum PSV (RI = PSV-MDV/PSV) was used as an additional parameter. All patients with suspected TRAS at Doppler US underwent renal graft arteriography, with TRAS being confirmed when there was a greater narrowing of the arterial lumen $>50 \%$, a significant stenosis diameter $[12,13]$.

We analyzed the following recipient variables: age at transplant; sex, weight, and height; body mass index $\left(\mathrm{BMI} ; \mathrm{kg} / \mathrm{m}^{2}\right)$; cause of primary kidney disease; systemic arterial hypertension ( $\mathrm{SAH}$ ) and diabetes (diabetes mellitus, DM); serology for cytomegalovirus; and serum levels of calcium, phosphorus, uric acid, cholesterol, LDLcholesterol, and triglyceride levels at the diagnosis of TRAS. We also assessed variables such as the type of donor (deceased or living), age, sex, renal function at the time of organ donation (creatinine), cause of death if the donor was deceased, and cold ischemia time.

The anastomosis site (common iliac artery, external iliac artery), number of graft arteries, lateralization of the kidney used in surgery (right or left), and presence of technical problems during surgery were evaluated. Regarding the post-transplant evolution, the following data were evaluated: induction therapy (anti-thymocyte rabbit immunoglobulin; Thymoglobulin or Basiliximab), initial immunosuppression (with or without prednisone), presence of delayed graft function (DGF), acute rejection diagnosis before stenosis, and diagnosis of cytomegalovirus infection.

Data directly related to TRAS, such as post-Tx time of diagnosis, with early TRAS being defined as that diagnosed within less than 90 days and late TRAS after 90 days or more, were also analyzed, in addition to serum creatinine levels, number of anti-hypertensive used, and mean systolic and diastolic blood pressure levels at TRAS diagnosis. All patients underwent Doppler US, and those suggestive of TRAS were compared with the gold standard, that is, arteriography. The comparison of the mean systolic and diastolic factors associated with stenosis of the renal graft artery blood pressure and creatinine levels, as well as the number of antihypertensive drugs before and after TRAS repair, was also performed. It was also assessed whether there was graft loss or death related to TRAS; clinical and laboratory data of patients with and without TRAS were taken to investigate the factors associated with stenosis development.

\section{Statistical Analysis}

At statistical analysis, continuous variables were expressed as mean \pm standard deviation and categorical variables as percentage or frequency. The comparison of continuous and categorical variables between the 2 patient groups (control and study) was carried out with the use of the Student $t$ test (for continuous variables) and Fisher exact test (for categorical variables), respectively. The correlation analysis between TRAS and possible risk factors was performed through Fisher exact test, $\chi^{2}$ test, and Pearson correlation. We calculated the adjusted measures of association (odds ratios, OR) with $95 \%$ confidence intervals (CI). Univariate and multivariate analyses were performed. In the univariate analysis, the variables that reached a probability value of $<15 \%$ were included in a multivariate logistic regression model. Descriptive values $<5 \%(P<.05)$ were considered statistically significant. Statistical analysis was performed with the use of SPSS 17.0 for Windows (SPSS Inc., Chicago, Ill, United States).

\section{RESULTS}

A total of 529 patients submitted to renal transplantation during the study period were assessed. Of these, 35 were excluded because of being transferred to other transplant units. All patients were evaluated for suspected stenosis of the renal artery graft by Doppler US. Thirty-seven patients were selected for having US Doppler results suggestive of TRAS, and 24 of these had TRAS confirmed by arteriography, as shown in Fig 1.

The prevalence of TRAS was $4.8 \%$ (24 patients), of which 23 had a deceased donor. Twelve patients $(50 \%)$ were men with a mean age of $46.7 \pm 13.5$ years (range, 17 to 78 years) and mean BMI of $23.3 \pm 3.6 \mathrm{~kg} / \mathrm{m}^{2}$ (range, 16.3 to $31.1 \mathrm{~kg} / \mathrm{m}^{2}$ ). The cause of chronic kidney disease was SAH 


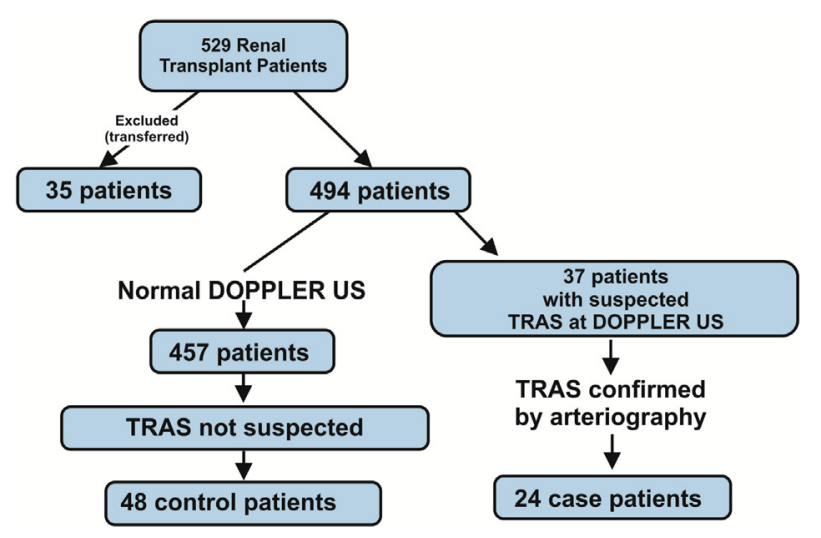

Fig 1. Characterization of the study population.

and DM in 6 patients (25\%) and glomerulopathies also in 6 patients $(25 \%)$.

Seropositivity for cytomegalovirus (immunoglobulin G) before the Tx was observed in 23 patients (95.8\%). Mean serum total calcium at diagnosis of TRAS was $10.2 \pm$ $1.4 \mathrm{mg} / \mathrm{dL}$ (range, 8.7 to $11.9 \mathrm{mg} / \mathrm{dL}$ ); phosphorus, $2.4 \pm$ $0.7 \mathrm{mg} / \mathrm{dL}$ (range, 1.8 to $3.1 \mathrm{mg} / \mathrm{dL}$ ); uric acid, $6.3 \pm 0.8 \mathrm{mg} /$ $\mathrm{dL}$ (range, 5.4 to $7.1 \mathrm{mg} / \mathrm{dL}$ ); LDL-cholesterol, $106.8 \pm 46$ $6 \mathrm{mg} / \mathrm{dL}$ (range, 44 to $152 \mathrm{mg} / \mathrm{dL}$ ); and triglycerides, $149.8 \pm$ $76.8 \mathrm{mg} / \mathrm{dL}$ (range, 54 to $242 \mathrm{mg} / \mathrm{dL}$ ). The recipient's mean creatinine level at time of TRAS diagnosis was $2.8 \pm 2.4 \mathrm{mg} / \mathrm{dL}$ (range, 0.9 to $9.9 \mathrm{mg} / \mathrm{dL}$ ). Table 1 shows the epidemiological and laboratory characteristics of the patients with TRAS compared with patients without TRAS. There was a statistically significant difference $(P=.04)$ between the mean creatinine levels of recipients with TRAS and the control group.

The donors had mean age and BMI of $34.6 \pm 15.7$ years (range, 10 to 61 years) and $24.5 \pm 3.1 \mathrm{~kg} / \mathrm{m}^{2}$ (range, 11 to
$62 \mathrm{~kg} / \mathrm{m}^{2}$ ), respectively, and 14 were men (58.3\%). The cause of death was traumatic brain injury in 13 patients $(54.2 \%)$ and cerebrovascular accident (stroke) in 9 patients $(37.5 \%)$.

The donor's right kidney was used for Tx in 14 patients $(58.3 \%)$, and arterial anastomosis occurred in the common iliac artery in $58.3 \%$ (14) patients with TRAS. The graft had 2 or more arteries in $54.2 \%$ of cases (13 patients). Technical difficulties were reported during surgery in only 6 patients in this group $(25 \%)$.

Thymoglobulin was the immunosuppression therapy induction used in 18 patients $(75.0 \%)$, and, at the maintenance, 20 patients $(83.3 \%)$ used a immunosuppressive regimen with corticosteroids. The prevalence of DGF was $66.7 \%$ (16 patients), acute rejection occurred in 3 patients $(12.5 \%)$, and no patient developed cytomegalovirus infection. The prevalence of SAH after Tx was 83.3\% (20 patients). When comparing the above-mentioned variables with the control group, there was a statistically significant association between TRAS and the graft that had 2 or more arteries $(P=.003)$. Mean total cold ischemia time was $20.8 \pm 6.3$ hours (range, 1.5 to 32.1 ) in the group with TRAS and $20.4 \pm 6.9$ (range, 0.6 to 41.6 ) hours in the group without TRAS (not a significant difference). Table 2 shows the characteristics associated with surgery and post-Tx period in patients with and without TRAS.

Regarding TRAS location, 15 were distal to the anastomosis $(62.5 \%)$ and 6 in the anastomosis $(25 \%)$. Early stenosis occurred in 13 patients $(54.2 \%)$, and, in 23 patients $(95.8 \%)$, stenosis occurred within less than 1 year after transplant. There was an association between TRAS and the mean number of anti-hypertensive drugs used when compared with the control group $(P=.001)$. The mean creatinine and systolic and diastolic blood pressure levels were higher in patients with TRAS when compared with the control group, with a statistically significant difference

Table 1. Demographic and Clinical-Laboratory Characteristics of the Groups With TRAS and Without TRAS

\begin{tabular}{|c|c|c|c|}
\hline $\mathrm{n}=72$ & With TRAS $(n=24)$ & Without TRAS $(n=48)$ & $P$ \\
\hline Recipient age, years & $46.7 \pm 13.5(17.0-78.0)$ & $46.0 \pm 12.9(15.0-73.0)$ & .82 \\
\hline \multicolumn{4}{|l|}{ Recipient sex } \\
\hline Male & $12(50 \%)$ & $26(54.2 \%)$ & .80 \\
\hline Female & $12(50 \%)$ & $22(45.8 \%)$ & \\
\hline Recipient BMI, kg/m² & $23.3 \pm 3.6(16.3-31.1)$ & $24.2 \pm 3.4(17.4-34.8)$ & .32 \\
\hline Donor age, years & $34.6 \pm 15.7(10.0-61.0)$ & $31.7 \pm 12.8(11.0-62.0)$ & .52 \\
\hline \multicolumn{4}{|l|}{ Donor sex } \\
\hline Male & $14(58.3 \%)$ & $36(75 \%)$ & .14 \\
\hline Female & $10(41.7 \%)$ & $12(25 \%)$ & \\
\hline Donor BMI, kg/m² & $24.5 \pm 3.1(19.0-33.2)$ & $24.6 \pm 3.7(13.7-32.1)$ & .88 \\
\hline Creatinine recipient, $\mathrm{mg} / \mathrm{dL}^{*}$ & $2.8 \pm 2.4(0.9-9.9)$ & $1.9 \pm 1.8(0.6-9.5)$ & .04 \\
\hline Creatinine donor, $\mathrm{mg} / \mathrm{dL}^{*}$ & $1.23 \pm 0.82(0.5-4.6)$ & $1.50 \pm 0.77(0.5-4.3)$ & .07 \\
\hline Calcium, mg/dL* & $10.2 \pm 1.4(8.7-11.9)$ & $10.3 \pm 1.3(8.0-12.0)$ & .63 \\
\hline Phosphorus, mg/dL* & $2.4 \pm 0.7(1.8-3.1)$ & $3.8 \pm 1.4(2.6-7.9)$ & .36 \\
\hline Uric acid, mg/dL* & $6.3 \pm 0.8(5.4-7.1)$ & $6.1 \pm 2.1(2.5-12.9)$ & .78 \\
\hline LDL-cholesterol, mg/dL* & $106.8 \pm 46.6(44.0-152.0)$ & $116.8 \pm 29.8(68.0-182.0)$ & .96 \\
\hline Triglycerides, mg/dL* & $149.8 \pm 76.8(54.0-242.0)$ & $149.5 \pm 77.1(42-332.0)$ & .65 \\
\hline
\end{tabular}

Abbreviations: TRAS, transplant renal artery stenosis; BMI, body mass index.

${ }^{*}$ At the diagnosis of TRAS compared with the control group, values are expressed as mean \pm standard deviation (minimum and maximum). 
Table 2. Factors Related to Surgery and Post-Transplantation in Groups of Patients With and Without TRAS

\begin{tabular}{lccc}
\hline \multicolumn{1}{c}{$\mathrm{n}=72$} & With TRAS $(\mathrm{n}=24)$ & Without TRAS $(\mathrm{n}=48)$ & $P$ \\
\hline Anastomosis & & & \\
$\quad$ Common iliac & $14(58.3 \%)$ & $17(35.4 \%)$ & .12 \\
$\quad$ External iliac & $10(41.7 \%)$ & $30(62.5 \%)$ & \\
No. of arteries & & & \\
$\quad<2$ & $11(45.8 \%)$ & $39(81.2 \%)$ & .003 \\
$\geq 2$ & $13(54.2 \%)$ & $9(18.8 \%)$ & \\
Organ & & & \\
$\quad$ Right kidney & $14(58.3 \%)$ & $22(45.8 \%)$ & .45 \\
$\quad$ Left kidney & $10(41.7 \%)$ & $26(54.2 \%)$ & \\
Technical difficulty & & & \\
$\quad$ Yes & $6(25.0 \%)$ & $5(10.4 \%)$ & .16 \\
$\quad$ No & $18(75.0 \%)$ & $43(89.6 \%)$ & \\
Induction & & & \\
$\quad$ Thymoglobulin & $18(75.0 \%)$ & $37(77.1 \%)$ & .48 \\
Simulect & $5(20.8 \%)$ & $11(22.9 \%)$ & \\
$\quad$ None & $1(4.2 \%)$ & - & \\
Corticoid & & & \\
$\quad$ Yes & $20(83.3 \%)$ & $32(66.7 \%)$ & .17 \\
$\quad$ No & $4(16.7 \%)$ & $16(33.3 \%)$ & \\
Delayed graft function & & & \\
$\quad$ Yes & $16(66.7 \%)$ & $26(54.2 \%)$ & .22 \\
No & $8(33.3 \%)$ & $22(45.8 \%)$ & \\
\hline
\end{tabular}

Abbreviation: TRAS, transplant renal artery stenosis.

(mean creatinine, $P=.04$; systolic and diastolic blood pressure, $P=.001$ ), as shown in Table 3 . The stenoses were associated with a number of arteries $\geq 2$ when compared with the group without TRAS $(P=.001)$. Table 4 shows the factors associated with early stenosis ( $<90$ days) and without TRAS.

The multivariate analysis included variables that reached a $P$ value <.15: sex and donor's cause of death, site of the arterial anastomosis, number of graft arteries, and number of anti-hypertensive drugs used. The number of the antihypertensive drugs was associated with higher risk of stenosis (OR, 17.0; CI, 4.1 to 70.4; $P=.001$ ). Multivariate analysis was also performed, comparing early with late stenoses regarding the number of graft arteries; 2 or more arteries was associated with increased risk of early stenosis, with an OR of 8.9 and CI of 1.4 to $56.6(P=.021)$, as shown in Table 5.

The mean time between the Tx and TRAS diagnosis was $89.9 \pm 86.3$ days (range, 2 to 431 days). The method used for TRAS diagnosis was Doppler US, which was suggestive of
Table 4. Factors Associated With Early TRAS

\begin{tabular}{lccr}
\hline $\mathrm{n}=61$ & With TRAS, $<90$ days & Without TRAS & $P$ \\
\hline $\begin{array}{l}\text { No. of anti-hypertensives } \\
<2\end{array}$ & $3(23.1 \%)$ & $34(70.8 \%)$ & .003 \\
$\geq 2$ & $10(76.9 \%)$ & $14(29.2 \%)$ & \\
$\quad$ Total & $13(100 \%)$ & $48(100 \%)$ & \\
No. of arteries & & & \\
$<2$ & $3(23.1 \%)$ & $39(81.3 \%)$ & .001 \\
$\geq 2$ & $10(76.9 \%)$ & $9(18.8 \%)$ & \\
Total & $13(100 \%)$ & $48(100 \%)$ & \\
\hline
\end{tabular}

Abbreviation: TRAS, transplant renal artery stenosis.

TRAS in 37 patients, being confirmed by arteriography in 24 patients.

All patients diagnosed with TRAS were treated with endovascular stent grafting through percutaneous transluminal coronary angioplasty. Only 1 patient lost the graft before stent implantation as the result of a bacterial infection in the renal allograft. After the stenosis treatment, the mean systolic and diastolic blood pressure and the lowest mean creatinine level were significantly lower when compared with the means measured before the procedure, as shown in Table 6. The mean number of antihypertensive drugs after TRAS repair was $1.6 \pm 1.1$ (range, 0 to 3 ), and there was a decrease in both the number and dose of anti-hypertensive drugs after stenosis repair in 13 patients $(54.2 \%)$.

Restenosis prevention occurred in 22 patients (91.7\%), with acetylsalicylic acid and clopidogrel being used in 21 patients $(87.5 \%)$, whereas only 1 patient received Warfarin $(4.1 \%)$. Five patients $(20.8 \%)$ had restenosis. One recipient lost the transplanted kidney because of infection and 1 recipient died of CVA, a cause unrelated to TRAS.

\section{DISCUSSION}

The observed prevalence of TRAS in our study was not high (4.8\%), and the mean time for TRAS diagnosis was 89.9 days. These data are similar to those found in the literature but high in relation to the Brazilian study, which shows a prevalence of $1.63 \%$ [18,30-32].

TRAS can be classified according to its location as preanastomosis, anastomosis, and post-anastomosis [16,22,32]. Occasionally, the narrowing can simultaneously affect multiple locations or even the entire vessel [22]. It can also be classified according to time of occurrence as early, that is,

Table 3. Factors Associated With TRAS

\begin{tabular}{lccc}
\hline & With TRAS $(\mathrm{n}=24)$ & Without TRAS $(\mathrm{n}=48)$ \\
\cline { 2 - 2 } \multicolumn{1}{c}{$\mathrm{n}=72$} & $\mathrm{SD}$ & $\mathrm{SD}$ & .003 \\
\hline Mean No. of arteries & $1.5 \pm 0.5(1-2)$ & $1.2 \pm 0.5(1-3)$ & .001 \\
No. of anti-hypertensives & $2.0 \pm 1.2(0-4)$ & $1.0 \pm 0.8(0-3)$ & .04 \\
Creatinine receptor & $2.8 \pm 2.4(0.9-9.9)$ & $1.9 \pm 1.8(0.6-9.5)$ & .001 \\
SBP & $147.1 \pm 23.7(100-208)$ & $127.8 \pm 15.2(100-175)$ & .001 \\
DBP & $86.6 \pm 13.0(53-110)$ & $77.6 \pm 9.4(60-101)$ & .010 \\
\hline
\end{tabular}

Abbreviations: TRAS, transplant renal artery stenosis; SD, standard deviation (minimum and maximum); SBP, systolic blood pressure; DBP, diastolic blood pressure. 
Table 5. Multivariate Analysis of Risk Factors for Transplant Renal Artery Stenosis

\begin{tabular}{lccc}
\hline & Odds Ratio & Confidence Interval & $P$ \\
\hline $\begin{array}{l}\text { No. of anti-hypertensives } \\
<2\end{array}$ & 17.0 & & \\
$\quad 2$ & & & \\
$\quad 2$ & & & \\
No. of graft arteries & & \\
$\quad<2$ & 8.9 & $1.4-56.6$ & .021 \\
$\geq 2$ & & & \\
\hline
\end{tabular}

within less than 3 months after transplant, or late [16]. According to Bruno et al [16], early anastomotic stenosis is probably related to trauma in the donor vessels during the capture of the organ or trauma in the recipient's vessels during attachment or suturing. Small lesions in the vessel intima or sub-intimal dissection of the vessel wall precede scars and vascular hyperplasia and, as a result, lumen narrowing or occlusion occurs $[16,18]$.

In our study, $54.2 \%$ had early TRAS and $95.8 \%$ of all stenoses occurred in less than 1 year, and there was a significant association of these stenoses with grafts that had 2 or more arteries, different from the study by Yildirim and Kucuk [28], which, perhaps because of the small number of patients, did not find any statistical significance when comparing patients with single or multiple arteries. AlHarbi et al [4] found $20 \%$ of early stenoses and $80 \%$ of them occurred in the first year, but they also had a small number of patients $(n=10)$ [4].

The incidence of multiple unilateral and bilateral renal arteries has been reported as being $23 \%$ and $10 \%$, respectively [29]. Transplanting a kidney with multiple arteries prolongs the warm ischemia time and increases rates of acute tubular necrosis and acute rejection, as well as being a challenge for the surgical team, but the use of these marginal organs is increasingly considered, given the growing number of recipients [29].

Audard et al [31] found 29 patients with stenosis (1.2\%), and all of them were late cases (mean, 41 months). Of these, $56.6 \%$ were proximal and $24 \%$ were distal to the anastomosis, unlike the stenoses observed in our study, in which most occurred distal to the anastomosis, in the donor artery $(62.5 \%)$. TRAS has a multifactorial etiology. Some factors, such as deceased donor, prolonged ischemia time, surgical technique with inadequate suture, arterial kinking, intimal injury during graft perfusion or during the transplantation, mechanical compression by vascular clamp during anastomosis, reactions to the suture material, excessive length of the renal artery, and twisting or tension in the suture site have been associated with increased rates of early TRAS, unlike late stenoses, which are associated with immunological factors such as cytomegalovirus infection, rejection of cyclosporine use, and recipient atherosclerosis $[1,2,4,19-22,28]$.

In our analysis, we compared the recipient's and the donor's sex and BMI, the type of induction, corticosteroid use, the presence of systemic arterial hypertension (SAH), DM, presence of DGF, acute rejection, levels of calcium, phosphorus, uric acid, LDL-cholesterol, and triglycerides at diagnosis of TRAS and cytomegalovirus infection among patients with and without TRAS, but there was no statistical significance. There was a tendency toward TRAS in patients whose donors had CVA as the cause of death and patients whose site of the anastomosis was in the common iliac artery $(58.3 \%)$, but there was no statistically significant difference when compared with the control group.

Etemadi et al [7] analyzed 700 patients and diagnosed late TRAS, 1 year after transplantation in 3 of them; they observed a significant association between TRAS and serum calcium, phosphorus, and LDL-cholesterol levels, with a trend toward statistical significance with uric acid, which suggests that these factors predispose to accelerated atherosclerosis and TRAS. Our study also assessed these data but found no statistical significance when compared with the control group; different from the above-mentioned study, most stenoses found in our research were early ones $(<3$ months after Tx).

Macia et al [19] found a significant association between TRAS and acute rejection through a retrospective review of 110 kidney transplants, in which $8.2 \%$ of their patients had TRAS associated with rejection. Wong et al [20], in a study of 77 cases of TRAS among 917 kidney transplants, found a significantly higher prevalence of TRAS in the acute cellular rejection group when compared with the control group matched for age, year of transplantation, sex, number of previous grafts, and corresponding class I human leukocyte antigen.

In 2006, Fernández-Nájera et al [30], in an analysis of 321 transplant patients, found 6 patients with TRAS, and, of these, 3 had acute rejection. In our analysis, acute rejection was diagnosed in 2 patients with TRAS $(12.5 \%)$ and in none of the patients without TRAS, a non-significant association, perhaps because of the small number of rejection cases.

The pathophysiology of TRAS is similar to that observed in native kidneys with ischemia to the parenchyma, resulting in renal dysfunction, increase in renin production, and subsequent activation of angiotensin II [33]. In our study, the use of 2 or more anti-hypertensive drugs was

Table 6. Blood Pressure and Serum Creatinine Levels Before and After Repair of Transplant Renal Artery Stenosis

\begin{tabular}{lccc}
\hline & Before Repair & After Repair & $P$ \\
\hline Serum creatinine, mg/dL & $2.8 \pm 2.4(0.9-9.9)$ & $1.6 \pm 1.8(0.5-9.9)$ & .001 \\
No. of anti-hypertensives & $2 \pm 1.2(0-4)$ & $1.6 \pm 1.1(0-3)$ & .042 \\
Systolic blood pressure & $147.1 \pm 23.7(100-208)$ & $128.0 \pm 18.1(100-170)$ & .002 \\
Diastolic blood pressure & $86.6 \pm 13.0(53-110)$ & $74.5 \pm 10.7(50-90)$ & .001 \\
\hline
\end{tabular}


significantly associated with stenosis; reducing the number of anti-hypertensive medications and blood pressure levels after the correction of the disease was also significant, showing the importance of stenosis screening in the group of patients who use a higher number of anti-hypertensives. There was also significant improvement in graft function, demonstrating the decrease in creatinine levels after the stenosis repair.

There was a trend toward the association of TRAS and the following variables, which reached a $P$ value of $<.15$ : donor sex (male), donor cause of death (CVA), and the site of the arterial anastomosis (common iliac artery), but there was no statistical significance. A number of antihypertensive drugs $\geq 2$ was associated with a 17-fold higher chance of developing TRAS when compared with the control group, supporting what is described in the literature, that is, that TRAS is a cause of resistant hypertension and graft dysfunction [1,7,12]. Multivariate analysis was also carried out, comparing the early and late stenoses regarding the number of graft arteries; it was observed that grafts with 2 or more arteries had an 8 -fold higher risk of early stenosis, which might be implicated as the cause (greater technical difficulty and smaller caliber vessels). The literature showed no similar multivariate analyses.

Doppler US is readily available, relatively inexpensive, and does not require radioactive markers, showing $87 \%$ to $94 \%$ sensitivity and $86 \%$ to $100 \%$ specificity for the diagnosis of TRAS; its only limitation is that results depend heavily on the experience and personal capacity of the ultrasonographist, because the identification of the renal artery is time-consuming and can be difficult in patients with multiple arteries [16,23,24,26,27,29]. In our study, the method used for diagnosing TRAS was the Doppler US, and patients with suspected TRAS by this method were submitted to arteriography for diagnostic confirmation.

The use of endovascular stent through percutaneous transluminal coronary angioplasty (PTCA) was the treatment of choice performed in $95.8 \%$ (23 patients) of TRAS cases, and only 1 patient lost the graft because of infection before the stenosis repair. According to Agroyannis et al [9], PTCA has been widely established as the treatment of choice for the treatment of TRAS, followed by stent implant, especially in ostial lesions, to provide protection against early occlusion or late restenosis. The surgical approach is recommended only when the lesion is inaccessible by PTCA or after an unsuccessful angioplasty [4,9].

Depending on the experience of each center and the type of lesion, PTCA can restore renal perfusion in $70 \%$ to $90 \%$ of the cases, although restenosis can occur in $20 \%$ of cases $[22,25]$. The restenosis rate in our study was $20.8 \%$ (5 patients), lower than that found by Audard et al [31], which was $27.5 \%$.

Patel et al [10] used a reduction of both serum creatinine and diastolic blood pressure $>15 \%$ with no change in antihypertensive drugs, or reduction $>10 \%$ in diastolic blood pressure, with a reduction in anti-hypertensive medication to define clinical success. These authors found a clinical success rate defined by improvement in blood pressure or creatinine levels, or both, of $82 \%$. In our study, there was a $13 \%$ reduction in mean systolic blood pressure and $10.4 \%$ reduction in mean diastolic blood pressure, in addition to a reduction in both the number and dose of anti-hypertensive drugs in $54.2 \%$ of cases after TRAS repair (13 patients; reduction of 2.69 to 1.61 anti-hypertensive drugs).

In $25 \%$ (6 patients), the number/dose of anti-hypertensive drugs remained unchanged, and $16.7 \%$ (4 patients) were not using anti-hypertensive drugs at TRAS diagnosis. Patients who did not have high blood pressure as suggestive criterion of stenosis or who did not change the dose/number of antihypertensive drugs after repair had renal dysfunction that significantly improved after stenosis repair, with mean creatinine level in this group before surgery of $2.1 \mathrm{mg} / \mathrm{dL}$ to $1.3 \mathrm{mg} / \mathrm{dL}$ after repair. The comparison between the mean systolic blood pressure and diastolic blood pressure before and after TRAS repair was significant, as well as the comparison between creatinine at the stenosis diagnosis and after its repair. This result discloses a clinical success rate of $100 \%$ among patients, either in blood pressure improvement or improvement in creatinine levels or both.

In the present study, there was a limitation because of the small number of patients diagnosed with TRAS in the study population, similar to other studies and the maximum number of 29 patients studied [7,10,21,27,30,31]. Another limitation was that all patients underwent a routine Doppler US after transplantation, but not at established periods and not with the intention of screening for TRAS; furthermore, they only underwent an arteriography, the gold standard for the diagnosis of stenosis, if the latter was suspected at the Doppler US. The importance of this study was to identify the prevalence of TRAS, which is not yet known in northeastern Brazil, and its associated factors when compared with those described in the literature.

\section{CONCLUSIONS}

The prevalence of TRAS found in the present study is consistent with the pattern described in the literature. Most stenoses occurred early ( $<3$ months), and the associated factors were the use of 2 or more anti-hypertensive drugs and grafts with multiple arteries. There was a significant decrease in systolic blood pressure and diastolic blood pressure, as well as in mean levels of serum creatinine after the stenosis repair; that is, if the condition goes undiagnosed, it may lead to continuous renal dysfunction, resistant hypertension, and possible renal graft deterioration. Further studies are required, or the continuation of this study, with a larger sample size, for a better assessment of factors associated with TRAS.

\section{REFERENCES}

[1] Aktas S, Boyvat F, Sevmis S, et al. Analysis of vascular complications after renal transplantation. Transplant Proc 2011;43: $557-61$. 
[2] Mendes WDS, Silva LF, Espinosa G, et al. Estenose Arterial nos Transplantes Renais. Rev Col Bras Cir 2005;32:237-43.

[3] Dimitroulis D, Bokos J, Zavos G, et al. Vascular complications in renal transplantation: a single-center experience in 1367 renal transplantations and review of the literature. Transplant Proc 2009;41:1609-14.

[4] Al-Harbi A, Chaudry T, Linjawi T, et al. Renal artery stenosis in renal transplantation presentation and management. Saudi J Kidney Dis Transpl 1998;9:22-6.

[5] Pillot P, Bardonnaud N, Lillaz J, et al. Risk factors for surgical complications after renal transplantation and impact on patient and graft survival. Transplant Proc 2012;44:2803-8.

[6] Leonardou P, Gioldasi S, Pappas P. Transluminal angioplasty of transplant renal artery stenosis: a review of the literature for its safety and efficacy. J Transplant 2011:1-5.

[7] Etemadi J, Rahbar K, Haghighi AN, et al. Renal artery stenosis in kidney transplants: assessment of the risk factors. Vasc Health Risk Manag 2011;7:503-7.

[8] Sharma S, Potdar A, Kulkarni A. Percutaneous transluminal renal stenting for transplant renal artery stenosis. Catheter Cardiovasc Interv 2011;77:287-93.

[9] Agroyannis B, Mourikis D, Tzanatos H, et al. Transplant renal artery stenosis: hypertension and graft function before and after angioplasty. J Hum Hypertens 2001;15:741-3.

[10] Patel NH, Jindal RM, Wilkin W, et al. Renal arterial stenosis in renal allografts: retrospective study of predisposing factors and outcome after percutaneous transluminal angioplasty. Radiology 2001;219:663-7.

[11] Buturović-Ponikvar J. Renal transplant artery stenosis. Nephrol Dial Transplant 2003;18(Suppl 5):74-7.

[12] Rengel M, Gomes-da-Silva G, Incháustegui L, et al. Renal artery stenosis after kidney transplantation: diagnostic and therapeutic approach. Kidney Int 1998;54(Suppl 68):99-106.

[13] Ruggenenti P, Mosconi L, Bruno S, et al. Post-transplant renal artery stenosis: the hemodynamic response to revascularization. Kidney Int 2001;60:309-18.

[14] Sanz YV, Lorente RRM, Berrocal FT, et al. Complicaciones vasculares en el transplante renal pediátrico: diagnóstico ecográfico. An Esp Pediatr 1999;50(3):263-8.

[15] Sun IO, Hong YA, Kim HG, et al. Clinical usefulness of 3-dimensional computerized tomographic renal angiography to detect transplant renal artery stenosis. Transplant Proc 2012;44:691-3.

[16] Bruno S, Remuzzi G, Ruggenenti P. Transplant renal artery stenosis. J Am Soc Nephrol 2004;15:134-41.

[17] Peregrin JH, Stríbrná J, Lácha J, et al. Long-term follow-up of renal transplant patients with renal artery stenosis treated by percutaneous angioplasty. Eur J Radiol 2008;66:512.
[18] Lopes JAM, Almeida CRJ, Hachul M, et al. Freqüência de estenose de artéria renal em 676 transplantes renais. Rev Assoc Med Bras 1998;44(3):210-3.

[19] Macia M, Paez A, Tornero F, et al. Post-transplant renal artery stenosis: a possible immunological phenomenon. J Urol 1991;145:251-2.

[20] Wong W, Fynn SP, Higgins RM, et al. Transplant renal artery stenosis in 77 patients: does it have an immunological cause? Transplantation 1996;61:215-9.

[21] Pouria S, State OI, Wong W, Hendry BM. CMV infection is associated with transplant renal artery stenosis. QJM 1998;91: $185-9$.

[22] Marini M, Fernandez-Rivera C, Gulias ICD, et al. Treatment of transplant renal artery stenosis by percutaneous transluminal angioplasty and/or stenting: study in 63 patients in a single institution. Transplant Proc 2011;43:2205-7.

[23] Henning BF, Kuchlbauer S, Böger CA, et al. Percutaneous transluminal angioplasty as first-line treatment of transplant renal artery stenosis. Clin Nephrol 2009;71:543-9.

[24] Polak WG, Jezior D, Garcarek J, et al. Incidence and outcome of transplant renal artery stenosis: single center experience. Transplant Proc 2006;38:131-2.

[25] Beecroft JR, Rajan DK, Clark TWI, et al. Transplant renal artery stenosis: outcome after percutaneous intervention. J Vasc Interv Radiol 2004;15:1407-13.

[26] Seratnahaei A, Shah A, Bodiwala K, Mukherjee D. Management of transplant renal artery stenosis. Angiology 2011;62: 219-24.

[27] Eufrásio P, Parada B, Moreira P, et al. Surgical complications in 2000 renal transplants. Transplant Proc 2011;43:142-4.

[28] Yildirim M, Kucuk HF. Outcomes of renal transplantations with multiple vessels. Transplant Proc 2011;43:816-8.

[29] Rajan DK, Stavropoulos SW, Shlansky-Goldberg RD. Management of transplant renal artery stenosis. Semin Intervent Radiol 2004;21:259-69.

[30] Fernández-Nájera JE, Beltrán S, Aparicio M, et al. Transplant renal artery stenosis: association with acute vascular rejection. Transplant Proc 2006;38:2404-5.

[31] Audard V, Matignona M, Hemeryb F, et al. Risk factors and long-term outcome of transplant renal artery stenosis in adult recipients after treatment by percutaneous transluminal angioplasty. Am J Transplant 2006;6:95-9.

[32] Becker BN, Odorico JS, Becker YT, et al. Peripheral vascular disease and renal transplant artery stenosis: a reappraisal of transplant renovascular disease. Clin Transplant 1999;13:349-55.

[33] Srinivas TR, Shoskes DA. Surgical complications after kidney transplantation. Curr Clin Urol 2001;15:288-9. 